\title{
Asteroid mass determination with the Gaia mission
}

\author{
Serge Mouret ${ }^{1}$, D. Hestroffer ${ }^{1}$ and F. Mignard ${ }^{2}$ \\ ${ }^{1}$ IMCCE/CNRS, UMR 8028, Paris observatory, Denfert-Rochereau 77, 75014 Paris, France \\ email: mouret@imcce.fr, Hestroffer@imcce.fr \\ ${ }^{2}$ OCA/Cassiopée/CNRS, UMR 6202, Nice observatory, Le Mont Gros, BP 4229, 06304 Nice \\ Cedex 4, France \\ email: francois.mignard@obs-nice.fr
}

\begin{abstract}
The main-belt asteroids are generally considered as the principal source for the nearEarth asteroids (NEAs). The ESA astrometric mission Gaia, due for launch in late 2011, will observe a very large number of asteroids $(\sim 300,000$ brighter than $\mathrm{V}=20)$, the majority lying in the main-belt and with an unprecedented positional precision (at the sub-milliarcsecond level). Such high precision astrometry will enable to derive direct measures of the masses of the largest asteroids of which their precise determination will be of utmost significance for the knowledge of physical properties of asteroids. The method for computing the masses is based on the analysis of orbital perturbations during close encounters between massive asteroids (perturbers) and several smaller minor planets (targets). From given criteria of close approaches selection, we give the list of asteroids for which the mass can be determined, and the expected precision of these masses at mission completion.
\end{abstract}

Keywords. astrometry; orbit determination; space missions; mass determination

\section{Introduction}

Two main sources for the near-Earth asteroids (NEAs) are generally recognized: the main-belt asteroids (MBAs the principal source) and nuclei of extinct comets. Gaia is an astrometric cornerstone mission of the European Space Agency. With a launch due in late 2011, Gaia will have a much more ambitious aim than its precursor Hipparcos: obtain a "3D census" of our galaxy with astrometric, photometric and spectroscopic observations. It will pinpoint its sources with an unprecedented positional precision (at the sub-milliarcsecond level for single observation) which will allow it to observe about 300,000 asteroids brighter than $\mathrm{V}=20$, the majority being in the main-belt. The high precision astrometry will enable us to considerably improve the orbits of almost all observed asteroids, yielding the masses of the largest one from mutual perturbations. The objective of this work is the determination of the masses of a small subset of minor planets, modeling the signature of the gravitational perturbation affecting smaller objects during close encounters. Not only does the method consider multiple close encounters with different targets, but it also simultaneously treats all perturbers together with their target asteroids. Here we explain our interest in the knowledge of the masses which when associated to the asteroids dimensions will probably bring precious information about the physical characteristics of main-belt asteroids and consequently of NEAs (albeit Gaia will probably not derive directly masses of NEA). We give a list of asteroids for which the mass can be determined, and the expected precision of these masses at mission completion from realistic simulations of the Gaia observations (such as geometry, time sequence and magnitude). 


\section{On the importance of masses}

Knowing the mass and size of an asteroid (the latter being from IRAS but also from Gaia) allows to determine its bulk density, which includes any space occupied by its pores. The calculation of the porosity, which enables us to estimate the part of void which makes up the asteroid, is of a great interest. It directly depends on the ratio between the bulk density of the asteroid and the grain density of an analog meteorite of similar composition. Knowing that during a collision, the internal structure of an asteroid acts upon the stress-wave propagation, which in turn, modifies its constitution, the porosity becomes a precious indicator of the collisional history of asteroids and their internal structure (Britt et al. 2002).

In addition, such measure of the bulk density will shed light on the relationship, if any, between the spectroscopic taxonomic class and the density, or possibly the porosity. Such progress could provide information on the origin and on the formation process of the solar system. If a well defined relationship can be evidenced, we could make reliable density estimates for objects of a known taxonomic characterization.

In the same way, the comparison between these densities for objects belonging to different taxonomic classes and the density of meteorites may strengthen the idea about the genetic relationship between different classes of meteorites and their supposed asteroid source (Zappalà \& Cellino 2002).

\section{The mass determination method}

The effect of a close encounter as well as is to modify the trajectory of the perturbed bodies or equivalently to change the initial conditions of its motion at some reference time. The observed minus calculated positions (O-C) for each observation of minor planets, which is expressed in Gaia longitude $\lambda$ projected over a given Great Circle, can be linearized and solved by the least squares technique,

$$
\mathbf{O}-\mathbf{C}=\mathbf{A}\left(\begin{array}{c}
\delta \mathbf{u}_{\mathbf{0}} \\
\delta \mathbf{m}_{\mathbf{p}}
\end{array}\right) \Rightarrow\left(\begin{array}{c}
\delta \mathbf{u}_{\mathbf{0}} \\
\delta \mathbf{m}_{\mathbf{p}}
\end{array}\right)=\left(\mathbf{A}^{t} \mathbf{A}\right)^{-1} \mathbf{A}^{t}(\mathbf{O}-\mathbf{C})
$$

where $\delta \mathbf{u}^{\mathbf{0}}=\left(\delta \mathbf{u}_{\mathbf{1}}^{\mathbf{0}}, \ldots, \delta \mathbf{u}_{\mathbf{n}}^{\mathbf{0}}\right)^{t}$ is the correction to initial conditions of the all $n$ asteroids with $\delta \mathbf{u}_{\mathbf{k}}^{\mathbf{0}}=\left(\delta x^{0}, \delta y^{0}, \delta z^{0}, \delta \dot{x}^{0}, \delta \dot{y}^{0}, \delta \dot{z}^{0}\right)$ the corrections on the position and velocity of asteroid $k$ at the initial time, and $\delta \mathbf{m}_{\mathbf{p}}=\left(\delta m_{1}, . ., \delta m_{p}\right)^{t}$ are the corrections on masses of the p perturbers.

The matrix A in equation (3.1) represents the partial derivatives of the longitudes $\lambda$ of the minor planets with respect to their initial parameters,

$[\mathbf{A}]_{i, j}=\sum_{q=1}^{3} \frac{\partial \lambda_{i}}{\partial x_{q}} \frac{\partial x_{q}}{\partial C_{j}^{0}} \quad$ where $\left\{\begin{array}{l}\mathbf{x}_{q=1, \ldots, 3}=(x, y, z) \\ \mathbf{C}=\left(\mathbf{u}_{1}^{0}, . ., \mathbf{u}_{n}^{0}, m_{1}, . ., m_{p}\right) \\ \mathbf{u}_{\mathbf{k}}^{0}=\left(x^{0}, y^{0}, z^{0}, \dot{x}^{0}, \dot{y}^{0}, \dot{z}^{0}\right) \text { for the } \mathrm{k}^{\text {th }} \text { asteroid } \\ \lambda \begin{array}{c}\text { is the vector made up of every observations } \\ \text { of all asteroids. }\end{array}\end{array}\right.$

The expression $\partial \lambda_{i} / \partial x_{q}$ is determined analytically, while the right-hand part of the decomposition $\partial x_{q} / \partial C_{j}$ is evaluated numerically. The latter is obtained by expressing the variations of the rectangular heliocentric coordinates $x_{q=1, . ., 3}$ (in an inertial frame) 
of each asteroid with respect to the adjustable parameters by integrating the variational equations simultaneously with the equations of motion (Herget 1968).

From equation (3.1), it is possible to give the precision $\sigma\left(\delta \mathbf{m}_{\mathbf{p}}\right)$ with which we will be able to determine the masses of perturbers $\mathbf{m}_{\mathbf{p}}$ knowing the precision of asteroid positions. If the normal matrix $\mathbf{A}^{t} \mathbf{A}$ is invertible, we obtain a relation between the variance-covariance matrix of the foreseen parameters $\operatorname{cov}\left(\delta \mathbf{u}^{\mathbf{0}}, \delta \mathbf{m}_{\mathbf{p}}\right)^{t}$ and the variancecovariance matrix $\operatorname{cov}(\mathbf{O}-\mathbf{C})$. Each line of the matrix $\mathbf{A}$ corresponding to an asteroid $k$ for an observation $i$ is weighted according to the error on the position $\sigma_{k, i}$ (depending on the magnitude) by a constant $\sigma_{0}$ and so, the measures of the weighted $(\mathbf{O}-\mathbf{C})$ have the same variance $\sigma_{0}^{2}$. In addition, we make the assumption that the measures on the positions are independent, consequently $\operatorname{cov}(\mathbf{O}-\mathbf{C})=\sigma_{0}^{2} \mathbf{E}$ and we obtain $(\mathbf{E}$ is a unit matrix):

$$
\operatorname{cov}\left(\delta \mathbf{u}^{\mathbf{0}}, \delta \mathbf{m}_{\mathbf{p}}\right)^{t}=\sigma_{0}^{2}\left(\mathbf{A}^{t} \mathbf{A}\right)^{-1}
$$

\section{The selection of target asteroids}

An important step for the mass determination is the selection of suitable target asteroids from which the gravitational signature of their perturbations by a larger asteroid is detectable. We restricted ourselves at this stage to the first 20,000 numbered asteroids for being potential targets, and considered that a potential perturber has a mass greater than $1 \times 10^{-13} M_{\odot}$ and belongs to the first 2,000 numbered asteroids. A close approach is considered meaningful if the impact parameter $b$ (the minimal distance between the two asteroid trajectories in the case where we do not take into account their mutual perturbations) is smaller than $0.2 \mathrm{AU}$ and the deflection angle $\theta$ greater than 5 mas estimated by:

$$
\tan \frac{\theta}{2}=\frac{G(m+M)}{v^{2} b}
$$

where $\mathrm{G}$ is the gravitational constant, $M$ the mass of the perturber, $m$ the mass of the target asteroid and $v$ the relative velocity of the encounter.

We selected the target asteroids from a systematic exploration of all the close approaches between perturbers and targets from 2011 to 2017 (see Table 1), so that the whole Gaia mission is covered.

\begin{tabular}{|c|c|c|c|}
\hline \multicolumn{4}{|c|}{ Number of } \\
\hline $\begin{array}{l}\text { perturbers } \\
\text { target asteroids }\end{array}$ & $\begin{array}{r}149 \\
2508\end{array}$ & $\begin{array}{l}\text { perturber and target } \\
\text { simultaneously }\end{array}$ & 54 \\
\hline
\end{tabular}

Table 1. Results of close approaches simulations

\section{Results}

Calculations have been performed by taking into account realistic simulation of the Gaia observations (such as geometry, time sequence and magnitude). First the number of perturbers with respect to the precision with which we will be able to determine their masses is given in Table 2. The results are expressed in terms of the relative precision $\sigma(m) / m$ where $m$ is the reference mass used in the simulation. Then, we give the ranking of the best relative precisions for the mass determination (Table 3). We can also see the number of targets for each mass determination, the number of close approaches for 
which one of the two characteristic periods (before or after the closest approach) will be out of the range of Gaia observations for the corresponding target asteroids, the formal precisions $\sigma(m)$, the standard masses $m$ taken for the perturbers and the relative precision.

Table 2. Number of the determined masses with respect to the relative precision

\begin{tabular}{lrll}
\hline \multicolumn{4}{c}{ Number of perturbers } \\
\hline & Total & 149 \\
\hline$\sigma(m) / m<0.1 \%$ & 2 & $\sigma(m) / m<20 \%$ & 61 \\
\hline$\sigma(m) / m<1 \%$ & 7 & $\sigma(m) / m<30 \%$ & 75 \\
\hline$\sigma(m) / m<10 \%$ & 46 & $\sigma(m) / m<50 \%$ & 81 \\
\hline
\end{tabular}

Table 3. The formal precisions on the best mass determination

\begin{tabular}{|c|c|c|c|c|c|c|}
\hline $\begin{array}{l}\text { Ast } \\
n^{\circ} \text { IAU }\end{array}$ & $\begin{array}{l}\text { teroid } \\
\text { name }\end{array}$ & $\begin{array}{c}\text { number of } \\
\text { target asteroids }\end{array}$ & $\begin{array}{c}\text { close approaches } \\
\text { not observed }\end{array}$ & $\begin{array}{c}\underset{\left[\mathrm{M}_{\odot}\right]}{\operatorname{sigma}} \sigma(m) \\
\end{array}$ & $\begin{array}{c}\operatorname{mass} m \\
{\left[\mathrm{M}_{\odot}\right]}\end{array}$ & $\sigma(m) / m$ \\
\hline 4 & Vesta & 562 & 214 & $4.22 \times 10^{-14}$ & $1.30 \times 10^{-10}$ & $0.033 \%$ \\
\hline 1 & Ceres & 336 & 109 & $1.68 \times 10^{-13}$ & $4.50 \times 10^{-10}$ & $0.037 \%$ \\
\hline 14 & Irene & 113 & 31 & $4.56 \times 10^{-14}$ & $2.60 \times 10^{-11}$ & $0.175 \%$ \\
\hline 27 & Euterpe & 137 & 39 & $2.54 \times 10^{-14}$ & $1.00 \times 10^{-11}$ & $0.254 \%$ \\
\hline 10 & Hygiea & 211 & 78 & $1.32 \times 10^{-13}$ & $4.70 \times 10^{-11}$ & $0.282 \%$ \\
\hline 52 & Europa & 134 & 73 & $9.46 \times 10^{-14}$ & $2.40 \times 10^{-11}$ & $0.394 \%$ \\
\hline 2 & Pallas & 23 & 2 & $5.78 \times 10^{-13}$ & $1.30 \times 10^{-10}$ & $0.445 \%$ \\
\hline 16 & Psyche & 165 & 70 & $1.41 \times 10^{-13}$ & $1.40 \times 10^{-11}$ & $1.010 \%$ \\
\hline 46 & Hestia & 21 & 5 & $1.22 \times 10^{-14}$ & $1.20 \times 10^{-12}$ & $1.020 \%$ \\
\hline 88 & Thisbe & 21 & 4 & $5.41 \times 10^{-14}$ & $5.00 \times 10^{-12}$ & $1.080 \%$ \\
\hline
\end{tabular}

\section{Conclusions}

This simulation taking into account 149 perturbers and some 2508 target asteroids gives very encouraging results since about 50 masses could be estimated with a precision better than $10 \%$. Keeping in mind that Gaia will actually observe about 300,000 asteroids, so that the number of close approaches is significantly increased, one can expect more mass determinations at that precision level. It is now planned to include these 300,000 asteroids in the simulations as well as more perturbers. Besides, we noticed that an important percentage $(>30 \%)$ of targets asteroids, for half of the perturbers, was found to be not observed by Gaia, either before or after the close approach time. So, a great part of information from the encounter between the perturber and target asteroids is lost and, a study of what could bring ground-based observations before and after the space mission seems to be essential.

\section{References}

Britt, D.T., Yeomans, D., Housen, K. \& Consolmagno, G. 2002, in: W.F. Bottke, A. Cellino, P. Paolicchi \& R.P. Binzel (eds.), Asteroids III, (University of Arizona Press, Tucson) p. 485 Zappalá, V. \& Cellino, A. 2002, in: O. Bienaymé \& C. Turon (eds.), GAIA: a european space project, (EAS Publications Series) vol. 2, p. 343

Herget, P. 1968, AJ 99, 225 